\title{
The future role of pharmacists in primary care
}

Life in primary care used to be relatively simple with GPs and pharmacists having well-defined roles and a boundary between them that was well recognised and generally respected by both. GPs prescribed and pharmacists dispensed medicines. There were, of course, always exceptions to these roles and blurring of the boundaries. Some GPs in remote and rural areas dispensed medicines, and pharmacists, while not actually prescribing, have always been happy to discuss patients' complaints with them and make recommendations for medicines to treat 'minor ailments' - a bit like a doctor. Pharmacists had an additional role of acting as error trappers for the occasional slip of the pen made by GPs. By and large, though, the two professions accepted their respective places in the primary care firmament.

However, this cosy clarity no longer prevails and this presents challenges to both professions. Pharmacists, in particular, have been seeking to redefine themselves and develop new and extended roles. Some pharmacists are now becoming independent prescribers and are allowed to prescribe without reference to a doctor. Even pharmacists who choose not to become prescribers are able to recommend a much wider range of overthe-counter medicines and, thereby, become involved in the treatment of a much wider range of conditions. They are facilitated in this by ongoing initiatives to deregulate medicines from prescriptiononly to pharmacy licensing status.

Pharmacists are also becoming increasingly involved in a variety of processes around medicines use known broadly as 'medicines management'; for example, pharmacists have been involved in the management of general practice repeat prescribing systems. ${ }^{1}$ They have also undertaken clinical medication reviews of older people in their own homes and in residential care and of patients with specific long-term conditions, such as heart failure. ${ }^{2-4}$

In England community pharmacists can now opt to provide a wide range of 'advanced and enhanced pharmaceutical services' which include medicines use reviews; anticoagulant monitoring services; disease-specific medicines management services; care home services; stop smoking services; supervised administration services (mainly for methadone); medicines assessment and compliance support services; prescriber support services; and screening services. ${ }^{5}$ The assumption of new roles by pharmacists is part of a global trend, with many of these new services falling under a broad rubric of 'cognitive pharmaceutical services'. ${ }^{6}$

Similar blurring of professional boundaries is happening in other areas of medicine and in and between other professions. Thus midwives increasingly do work traditionally done by obstetricians, nurses the work of doctors and social workers, conveyancers the work of solicitors, solicitors the work of barristers, and so on. Sociologists speculate that this erosion of the traditional boundaries of professions is driven by diverse social pressures, such as globalisation, marketisation, managerialism, and consumerism. ${ }^{7}$

Since pharmacists have acquired the capacity to recommend or prescribe more medicines without reference to a doctor and have taken on these new roles, their effectiveness has come under some scrutiny. ${ }^{8}$ Thus, Davis et al in this month's $B J G P$ assess the impact of the availability of over-the-counter topical chloramphenicol on the appropriateness of its use. ${ }^{9}$ They found that over-the-counter availability was associated with an overall increased in sales, which differs from evidence published (coincidentally around the same time as the medicine was deregulated) showing that topical antibiotics were not very effective in acute conjunctivitis. GPs, however, were shown to have heeded the evidence, albeit to a rather limited extent.

Given that it has taken a long time and a lot of effort to start to get GPs to prescribe antibiotics in a more evidence-based manner, this would seem to be an inappropriate time at which to liberalise access to these drugs. ${ }^{10}$ Similar concerns have been expressed in the past about whether pharmacists are applying evidence to the dispensing of over-the-counter medicines; for example, topical antifungals in vaginal candidiasis. ${ }^{11}$ Watson et al described an educational initiative which presented an evidence-based guideline accompanied by educational outreach visits or continuing-professional-education sessions to improve the application of evidence by pharmacists, but its implementation had a disappointing lack of impact. $^{12}$

The promotion of advanced and enhanced pharmaceutical services is partly based on evidence, mostly from the US, suggesting that they can be effective, at least in economic terms. ${ }^{13}$ However, recent reviews that have sought to quantify the benefit of medication reviews conducted in primary care by pharmacists or others, in terms of adverse effects, hospital admission, and deaths, have been disappointing. ${ }^{14,15}$ Royal et al looked at studies in primary care aimed at reducing medication-related adverse events that result in morbidity, hospital admissions, and/or mortality. ${ }^{14}$ While they found that pharmacist-led interventions in a meta-analysis of 17 studies appeared to be effective in reducing hospital admissions $(\mathrm{OR}=0.64, \mathrm{Cl}=0.43$ to 0.96$)$, this perception disappeared when analysis was restricted to the nine randomised controlled trials involving over 10000 patients (OR = $0.92, \mathrm{Cl}=0.81$ to 1.05$)$. Holland et al looked at pharmacist-led medication reviews in older people only, and found no significant benefit in terms of emergency hospital admission or mortality. ${ }^{15}$ However, they did note a possible decrease in the numbers of drugs prescribed and positive effects on other intermediate outcomes, such as drug knowledge, adherence, and drug storage.

In a future issue of the BJGP, the RESPECT trial team report on a trial designed to determine the benefits of pharmacists' interventions in the medicines use of older patients. ${ }^{16}$ Their model of pharmaceutical care was one that involved 
collaboration between GPs and community pharmacists trained in pharmaceutical care. Pharmacists reviewed the medicines prescribed to older patients (aged over 75 years), living at home, and whom were receiving five or more medicines on repeat prescription. ${ }^{17}$ The pharmacists discussed medicine-related problems with the GP and together they devised, implemented, and monitored pharmaceutical care plans. The primary outcome measure used was an anglicised version of the Medication Appropriateness Index (MAl)..$^{18}$ Other outcomes included measures of quality of life (Short Form-36 Health Survey; SF-36) and health utility (EQ-5D). No benefit for this model of pharmaceutical care was shown in terms of any of these outcome measures.

These findings are difficult to explain given the ample evidence there is that misuse of medicines, particularly in older people, is associated with morbidity and mortality, and the obvious role for pharmacists to address this issue. A hint of what might be going wrong is provided by a qualitative study which examined transcripts of consultations between pharmacists and older patients in a trial of pharmacist-led pharmaceutical care for heart failure..$^{19}$ In this study it was found that advice from pharmacists was usually quite didactic and was often resisted or rejected by patients for whom the advice was largely unsolicited and not related to information sought by them.

There are also methodological problems in ascertaining the benefits of pharmaceutical care. Measures such as mortality or hospital admission rates may be subject to so many other factors, as to be a somewhat unfair measure. Measures of prescribing appropriateness, such as the MAI, are also limited in their capacity to reflect all aspects of prescribing, and may be insensitive to small but important changes in the patient's drug regimen. ${ }^{20}$ Likewise, broad-based quality of life measures, such as the SF-36, may not detect important effects of interventions in individual patients. ${ }^{21}$

Studies that appear to demonstrate benefits from pharmaceutical care more clearly tend to focus on more restricted clinical indications (diabetes, hypertension, asthma), which have shown benefits in terms of intermediate or proxy clinical outcomes (glycated hemoglobin, blood pressure, peak expiratory flow rate), rather than morbidity or mortality, and have involved pharmacists dedicated more exclusively to the cognitive service role.

Establishing clinically significant benefits across the range of pharmaceutical care which might be provided by community pharmacists would require enormous studies. However, the recurring failure of well-conducted clinical studies to demonstrate an unequivocal benefit for pharmacists in their new pharmaceutical care role does constitute something of a problem for the profession. Ford, responding to a $B M J$ editorial highlighting the same difficulty, suggests that the problem lies with the position of the pharmacist as still rather semi-detached from the primary care team, and his or her interventions, particularly cognitive interventions, coming too late in the prescribing-dispensing sequence..$^{22}$ It may be an overstatement to assert that primary care pharmacy is in a crisis but it is certainly facing something of a dilemma.

While there is an understandable desire to move away from the traditional subservient role in relation to the medical profession, there remains a substantial challenge facing the pharmacy profession to attest its selfdeclared professional role of providing optimal health care for patients. Effective delivery of this function may require a more significant move into the fold of the primary care team - possibly even abandoning the shop - and learning new ways of interacting with patients and medical colleagues.

\section{CP Bradley}

Professor of General Practice,

University College Cork, Cork, Ireland.

\section{Provenance}

Commissioned; not peer reviewed.

\section{REFERENCES}

1. Zermansky AG, Petty DR, Raynor DK, et al. Randomised controlled trial of clinical medication review by a pharmacist of elderly patients receiving repeat prescriptions in general practice. BMJ 2001; 323(7325): 1340-1343.

2. Holland R, Lenaghan E, Harvey I, et al. Does home based medication review keep older people out of hospital? The HOMER randomised controlled trial. BMJ 2005; 330(7486): 293.

3. Zermansky AG, Alldred DP, Petty DR, et al. Clinical medication review by a pharmacist of elderly people living in care homes - randomised controlled trial. Age Ageing 2006; 35(6): 586-591.

4. Holland R, Brooksby I, Lenaghan E, et al. Effectiveness of visits from community pharmacists for patients with hear failure: HeartMed randomised controlled trial. BMJ 2007; 334(7603): 1098

5. Noyce PR. Providing patient care through community pharmacies in the UK: policy, practice, and research. Ann Pharmacother 2007; 41(5): 861-868.

6. Academy of Managed Care Pharmacy. Pharmacists cognitive services. Alexandria, VA: AMCP, 2007.

7. Lane $\mathrm{K}$. The plasticity of professional boundaries: a case study of collaborative care in maternity services. Health Sociol Rev 2006; 15(4): 341-352.

8. Ballantyne $P$. The role of pharmacists in primary care. $B M J$ 2007; 334(7603): 1066-1067.

9. Davis H, Mant D, Scott C, et al. Relative impact of clinical evidence and over-the-counter prescribing on topical antibiotic use for acute infective conjunctivitis. $\mathrm{Br} J \mathrm{Gen}$ Pract 2009; 59(569): 897-900.

10. Meropol S, Chen Z, Metlay JP. Reduced antibiotic prescribing for acute respiratory infections in adults and children. Br J Gen Pract 2009; 59(567): e321-328.

11. Ferris DG, Nyirjesy P, Sobel JD, et al. Over-the-counter antifungal drug misuse associated with patient-diagnosed vulvovaginal candidiasis. Obstet Gynecol. 2002; 99(3): 419-425.

12. Watson MC, Bond CM, Grimshaw JM, et al. Educational strategies to promote evidence-based community pharmacy practice: a cluster randomised controlled trial (RCT). Fam Pract 2002; 19(5): 529-536.

13. Perez A, Doloresco F, Hoffman JM, et al. ACCP: economic evaluation of clinical pharmacy services: 2001-2005. Pharmacotherapy 2009; 29(1): 128.

14. Royal S, Smeaton L, Avery AJ, et al. Interventions in primary care to reduce medication related adverse events and hospital admissions: systematic review and metaanalysis. Qual Saf Health Care 2006; 15(1): 23-31.

15. Holland R, Desborough J, Goodyear L, et al. Does pharmacist-led medication review help to reduce hospital admissions and deaths in older people? A systematic review and meta-analysis. Br J Clin Pharmacol 2008; 65(3): 303-316.

16. RESPECT trial team. Effectiveness of shared pharmaceutical care for older patients: RESPECT trial findings. Br J Gen Pract 2009. Advance online publication. DOI: 10.3399/bjgp09X473295.

17. Wong I, Campion P, Coulton S, et al. Pharmaceutical care for elderly patients shared between community pharmacists and general practitioners: a randomised evaluation. RESPECT (Randomised Evaluation of Shared Prescribing for Elderly people in the Community over Time). BMC Health Serv Res 2004; 4(1): 11

18. Hanlon JT, Schmader KE, Samsa GP, et al. A method for assessing drug therapy appropriateness. J Clin Epidemiol 1992; 45(10): 1045-1051.

19. Salter C, Holland R, Harvey I, Henwood K. 'I haven't even phoned my doctor yet.' The advice giving role of the pharmacist during consultations for medication review with patients aged 80 or more: qualitative discourse analysis. $B M J$ 2007; 334(7603): doi: 10.1136/bmj.39171.577106.55.

20. Barber N, Bradley C, Barry C, et al. Measuring the appropriateness of prescribing in primary care: are current measures complete? J Clin Pharm Ther 2005; 30(6): 533-539.

21. Carr AJ, Higginson IJ. Are quality-of-life measures patient centred? BMJ 2001; 322(7298): 1357-1360.

22. Ford $\mathrm{S}$ Twelve years later ... [Rapid response]. http://www.bmj.com/cgi/eletters/334/7603/1066\#167299(a ccessed 11 Nov 2009).

DOI: 10.3399/bjgp09X473105

ADDRESS FOR CORRESPONDENCE

\section{CP Bradley}

University College Cork, Department of General Practice, Distillery House, North Mall, Cork, Ireland. E-mail: c.bradley@ucc.ie 